\title{
Using PIV to measure granular temperature in saturated unsteady polydisperse granular flows
}

\author{
Nicoletta Sanvitale $^{1}$ - Elisabeth T. Bowman ${ }^{1}$
}

Received: 22 July 2015 / Published online: 1 July 2016

(C) The Author(s) 2016. This article is published with open access at Springerlink.com

\begin{abstract}
The motion of debris flows, gravity-driven fast moving mixtures of rock, soil and water can be interpreted using the theories developed to describe the shearing motion of highly concentrated granular fluid flows. Frictional, collisional and viscous stress transfer between particles and fluid characterizes the mechanics of debris flows. To quantify the influence of collisional stress transfer, kinetic models have been proposed. Collisions among particles result in random fluctuations in their velocity that can be represented by their granular temperature, T. In this paper particle image velocimetry, PIV, is used to measure the instantaneous velocity field found internally to a physical model of an unsteady debris flow created by using "transparent soil"-i.e. a mixture of graded glass particles and a refractively matched fluid. The ensemble possesses bulk properties similar to that of real soil-pore fluid mixtures, but has the advantage of giving optical access to the interior of the flow by use of plane laser induced fluorescence, PLIF. The relationship between PIV patch size and particle size distribution for the front and tail of the flows is examined in order to assess their influences on the measured granular temperature of the system. We find that while PIV can be used to ascertain values of granular temperature in dense granular flows, due to increasing spatial correlation with widening gradation, a technique proposed to infer the true granular temperature may be limited to flows of relatively uniform particle size or large bulk.
\end{abstract}

This article is part of the Topical Collection on Micro origins for macro behavior of granular matter.

\footnotetext{
Nicoletta Sanvitale

n.sanvitale@sheffield.ac.uk

1 Department of Civil and Structural Engineering, University of Sheffield, Sir Frederick Mappin Building, Mappin St, Sheffield S1 3JD, UK
}

Keywords Granular flow · Particle image velocimetry · Debris flows · Granular temperature

\section{Introduction}

Debris flows are highly dangerous gravity-driven mass flows of soil, rock and water [1]. The motion of such flows may be considered in the light of granular flow theory which has been developed to describe the behaviour of ensembles of particles undergoing shearing motion under gravity-encompassing pseudo-static, frictional, collisional and kinetic regimes [2]. While theories of granular flow have been developed by focusing on steady, monodisperse systems, debris flows are far more complex, and may exhibit behaviour across the whole spectrum, even within a single flow event [3].

Particle sizes within debris flows can range over six orders of magnitude. Due to particle size segregation, the front of a debris flow surge is usually unsaturated as the larger particles focus forward and act as a frictional "break" to the fluidised body and tail of the flow [4]. The erosion of the bed along the path of a debris flow also has been found to occur more at the front [5], which suggests that the motion of the largest particles is particularly important to the erosion mechanism. The highly dilute, saturated tail, which is dominated by fines, may predispose the channel to further erosion by the gradual transfer of moisture from the flow into the bed, preconditioning it to subsequent flow surges. These elements point to the consideration of the diverse roles that particles of different size may play within a given flow-and that these can be as important as their ensemble behaviour. These unique mechanical characteristics have led to a particular interest in understanding the stress transfer behaviour between different elements of the flow. 
The majority of work on stress transfer mechanics in particulate flows has considered uniform, steady systems. With the exception of some work on dry 2D systems of bidisperse materials [6], numerical work has focused almost solely on spherical mono-disperse particle flows $[2,7,8]$, while physical tests have generally examined flows of regular spherical or sub-rounded particles [9-14]. Regimes of behaviour describing collision-dominated, friction-dominated or fluid viscous-dominated flows are generally determined according to non-dimensional numbers such as Bagnold and Savage [15-17]. The dimensionless groups incorporate for example, particle size, shear rate and fluid viscosity in their determination and calculated flow regimes are based on particular thresholds that have been determined from physical experiments on single-sized particle flows.

Work on monodisperse materials has been transferred directly into attempts to understand debris flows, without considering that such flows are extremely polydisperse, with typical values of coefficient of uniformity $\mathrm{C}_{\mathrm{U}}$ (ratio of the diameter of $60 \%$ finest by weight $\left(\mathrm{D}_{60}\right)$ to that of $10 \%$ finest by weight $\left.\left(\mathrm{D}_{10}\right)\right)$ of the order of 1000 [3]. To do this, the mean particle size $\left(\mathrm{D}_{50}\right)$ is generally taken as being representative of the flow as a whole and shear rates are determined according to a mean front velocity or surface velocity [16,18-21]. At least in geomechanics terms, $\mathrm{D}_{50}$ has little physical meaning while, as we see here, shear rate may have significant variation with depth.

Collisional stress transfer between particles results in random fluctuations in the velocity of the particles. Kinetic theory considers such random fluctuations to be represented by the "granular temperature" T [22], in analogy to gas physics [23]. For a monodisperse system of spheres (3D) or disks (2D), the granular temperature can be measured by tracking the centres of the particles and assuming collision takes place when the centres become equal to twice the radius of the particles. For dispersed systems, methods that use particle tracking, such as Particle Tracking Velocimetry, PTV, may be most suitable to physically measure granular temperature. However, for dense fast flows comprising irregular particles, collisions cannot be ascertained by direct tracking. Particle image velocimetry, PIV, has been presented as an effective solution for the measurement of an instantaneous velocity field for such flows over a broad range of spatial scales. In this way, the measurement of granular temperature can be related to the average of the squared particle fluctuation velocity [24-26].

Preliminary work was carried out to examine the use of PIV to measure the granular temperature interior to a relatively uniform (though not monodisperse) saturated dense granular flow [27] using transparent "soil" and a refractively matched fluid, coupled with Plane Laser Induce Fluorescence, PLIF. The use of refractive index matching (RIM) and PLIF allowed access to the interior of the flow, away from the influence of sidewalls [28], where otherwise, frictional resistance can dominate and give unrepresentative measurements of velocity fields [29]. This paper extends this preliminary work to mixtures consisting of well-graded solids. In doing so we examine some issues associated with the PIV technique as applied to granular flow related to the behaviour of the interior of polydisperse, unsteady and segregating flows over rigid beds.

\section{Methods}

\subsection{Small-scale flume arrangement}

The experiments were conducted using a small-scale flume equipped with a hopper at the top of the slope which held the material before its release (Fig. 1). A curved chute extending from the hopper, led to a $2 \mathrm{~m}$ long and $150 \mathrm{~mm}$ wide straight channel with transparent sidewalls and a frictional base set at an angle of $24.5^{\circ}$. The glass bottom of the slope was artificially roughened over a length of $1 \mathrm{~m}$. A $1.5 \mathrm{~mm}$ thick $532 \mathrm{~nm}$ laser light sheet was allowed to pass through the bottom to illuminate the flowing material at a distance of $35 \mathrm{~mm}$ from the transparent sidewall. The illuminated plane was located about $200 \mathrm{~mm}$ upstream of the channel outlet. For each test, the mixture consisting of glass particles and a refractively matched fluid were at first gently agitated to ensure an initially unconsolidated response, and then were released from the hopper via a trap door. Plane laser induced fluorescence, PLIF, was then implemented to track the flow internally. A high speed camera recorded the flow behaviour in the illuminated plane at 1100 frames per second with a resolution of $1280 \times 256$ pixels. The experimental set up is described in more detail in [28].

\subsection{Mixture properties and PLIF}

The purpose of these experiments was to investigate the internal behaviour of saturated granular free-surface flows. To this end, we applied an optical method which relies on the use of transparent materials by a careful refractive index matching (RIM) of the solid and fluid phase. A number of different pairings of transparent particles and associated fluids are suitable, provided they have compatible refractive indices [30]. Particles made from borosilicate glass, Duran ${ }^{\circledR}$, manufactured by Schott, and a hydrocarbon immersion liquid specifically produced by Cargille Laboratories to closely match the refractive index of Duran ${ }^{\circledR}$ were used (Table 1). In this work the physical properties of the experimental solids and fluid were required to compare closely with those of real debris flow materials [16]. The relative densities of the glass and the oil were slightly less than those of soil and water, although the ratio of the densities were the same at 2.65, how- 
Fig. 1 Apparatus employed in the tests

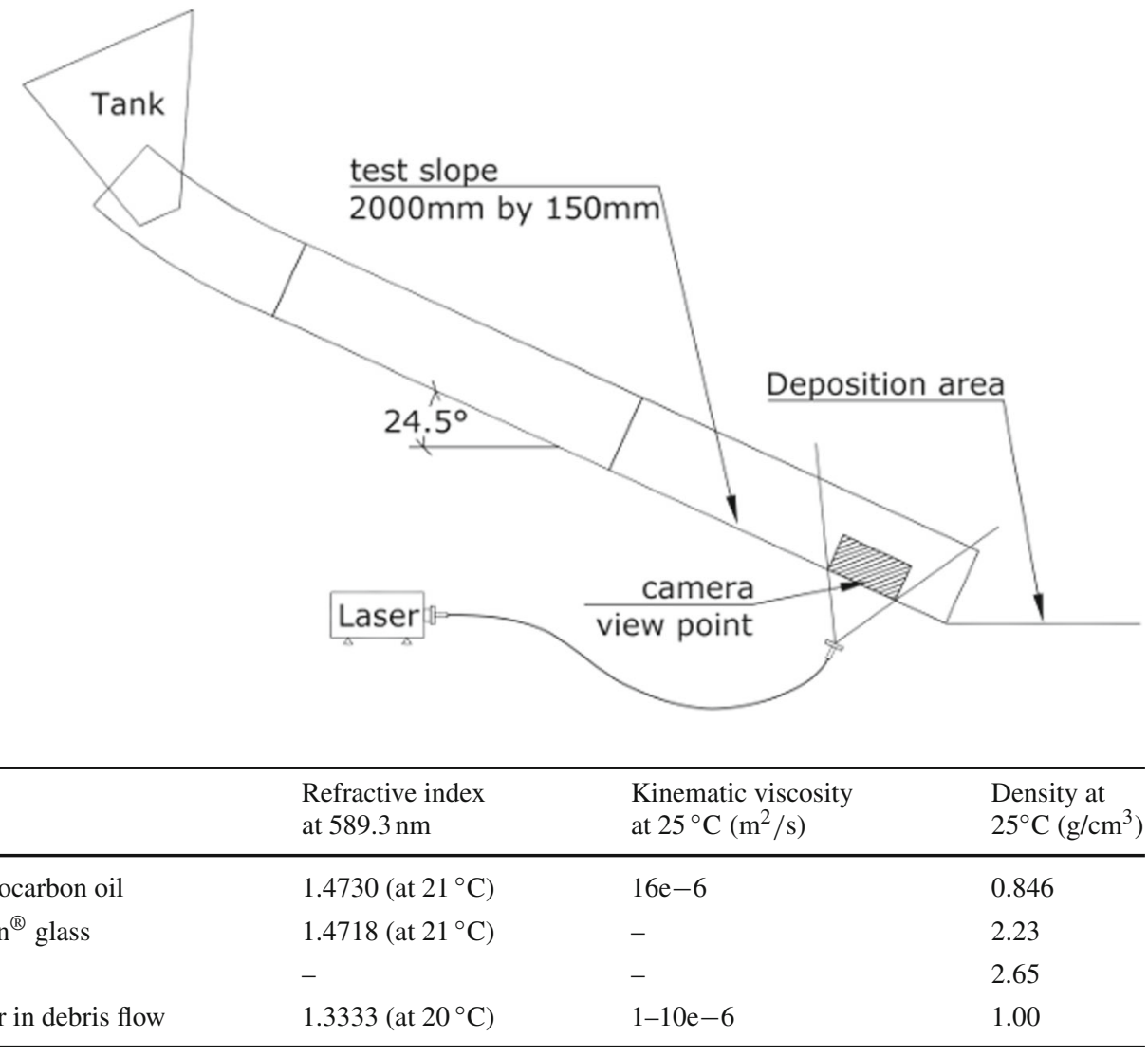

\begin{tabular}{llll}
\hline & $\begin{array}{l}\text { Refractive index } \\
\text { at } 589.3 \mathrm{~nm}\end{array}$ & $\begin{array}{l}\text { Kinematic viscosity } \\
\text { at } 25^{\circ} \mathrm{C}\left(\mathrm{m}^{2} / \mathrm{s}\right)\end{array}$ & $\begin{array}{l}\text { Density at } \\
25^{\circ} \mathrm{C}\left(\mathrm{g} / \mathrm{cm}^{3}\right)\end{array}$ \\
\hline Hydrocarbon oil & $1.4730\left(\right.$ at $\left.21^{\circ} \mathrm{C}\right)$ & $16 \mathrm{e}-6$ & 0.846 \\
Duran ${ }^{\circledR}$ glass & $1.4718\left(\right.$ at $\left.21^{\circ} \mathrm{C}\right)$ & - & 2.23 \\
Soil & - & - & 2.65 \\
Water in debris flow & $1.3333\left(\right.$ at $\left.20^{\circ} \mathrm{C}\right)$ & $1-10 \mathrm{e}-6$ & 1.00 \\
\hline
\end{tabular}

Table 1 Solid and fluid properties ever, the fluid viscosity of hydrocarbon oil was $\sim 20$ times greater than that of water (see Table 1). It was considered to be important to replicate the development and dissipation of non-hydrostatic pore pressure as occurs for debris flows. To do this, accounting for the increased fluid viscosity and slight density effects, the glass particles were scaled up four times with respect to a prototype particle size distribution which was termed "PSD9" in previous research [28]. This resulted in a similar hydraulic conductivity and was deemed a suitable replicate for use. Duran ${ }^{\circledR}$ glass particles with irregular shapes were used in order to mimic the friction and rotational resistance between soil grains (Fig. 2). The fluid included a small amount of fluorescent dye (Nile Red) so that when a laser was shone through the glass-oil system, the oil would show up bright and the particles dark, enabling a 2D planar slice of the flow to be recorded away from the sidewalls. In these experiments this measurement was taken at $35 \mathrm{~mm}$ into the $150 \mathrm{~mm}$ wide flow.

\subsection{Test conditions}

For all experiments, a mixture was prepared with $12 \mathrm{~kg}$ of borosilicate (Duran) glass particles and fluid to produce a mean solids concentration of 0.57 (porosity of 0.43 ). Three particle size distributions, PSDs, were used (Fig. 3) to examine the influence of a change in the coefficient of uniformity
$\mathrm{C}_{\mathrm{U}}=\mathrm{D}_{60} / \mathrm{D}_{10}$ around a particular mean particle size $\mathrm{D}_{50}$ of $7.1 \mathrm{~mm}$, where $\mathrm{D}_{x}$ denotes the percentage passing by mass. Materials termed "PSD9up" and "PSD11up" were wellgraded, with coefficient of uniformity equal to 20.2 (denoted to one significant figure, " $\mathrm{C}_{\mathrm{U}}=20$ ") and 9.8 (" $\mathrm{C}_{\mathrm{U}}=10$ ") respectively, whereas "PSD16up" was comparatively uniform with $\mathrm{C}_{U}=3.3$ ("C $\mathrm{C}_{U}=3$ "). For each grading, two experiments were carried out to ensure repeatability. In general, it should be noted that the majority of experimental work on granular debris flow is carried out on materials with approximately $\mathrm{C}_{\mathrm{U}}=1$ (i.e. monodisperse), therefore the behaviour of PSD16up $\left(\mathrm{C}_{U}=3\right)$ most closely replicates this here. In contrast, most debris flows have (scaled) PSDs that most closely resemble PSD9up $\left(\mathrm{C}_{U}=20\right)$, although this would be the lower bound of their polydispersity.

\subsection{Image processing}

The internal velocity field of the granular flows was obtained via a Particle Image Velocimetry approach, using GeoPIV software [31]. GeoPIV calculates the displacement field within a plane via a series of images taken over the course of deformation. It does this by tracking the image texture (i.e. the spatial variation of brightness) of sub-regions or "patches" of the original image in subsequent frames. The original version of GeoPIV was modified to examine granular 

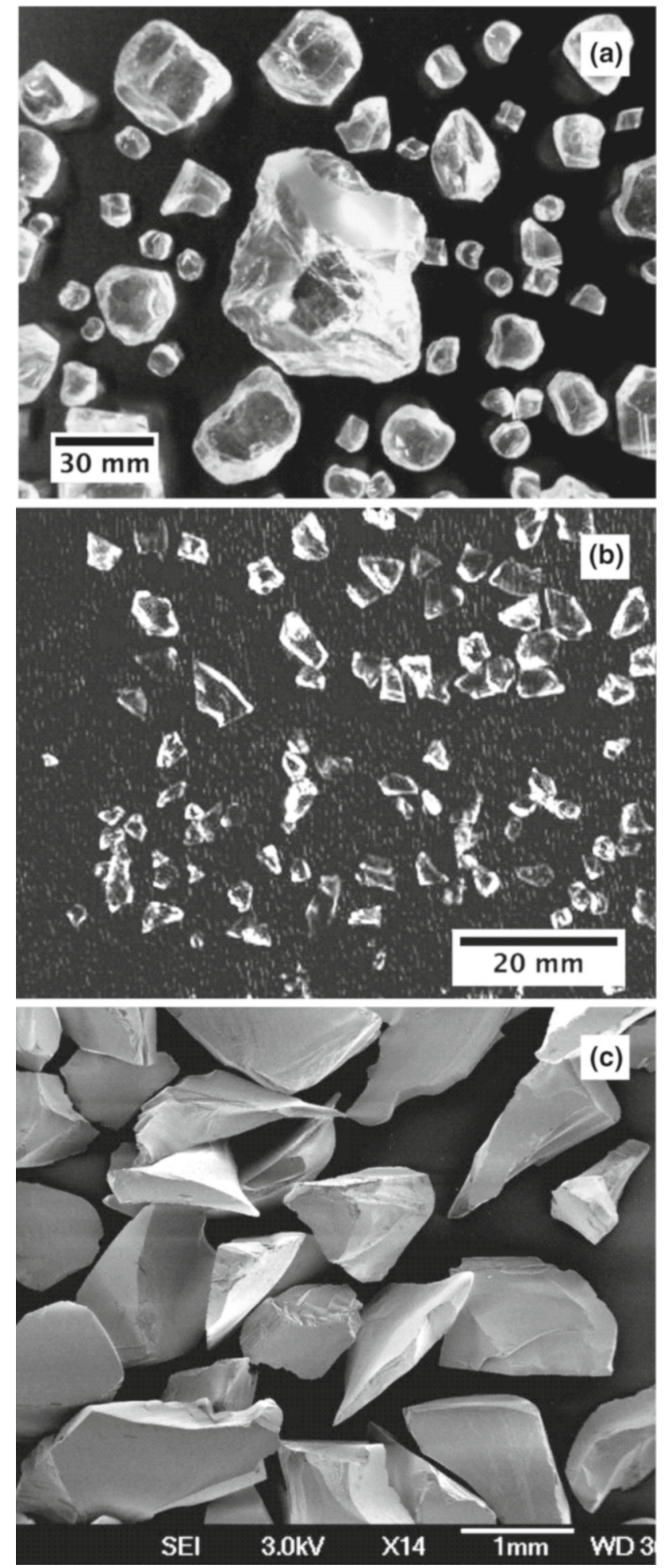

Fig. 2 Glass particles used as transparent solid. a Coarse grains; b particles finer than $4 \mathrm{~mm}$; c SEM image of $600 \mu \mathrm{m}$ particles

flow behaviour by supporting a static mesh of interrogation patches with position and geometry fixed in all images [32]. The meshes were composed of a single column of square

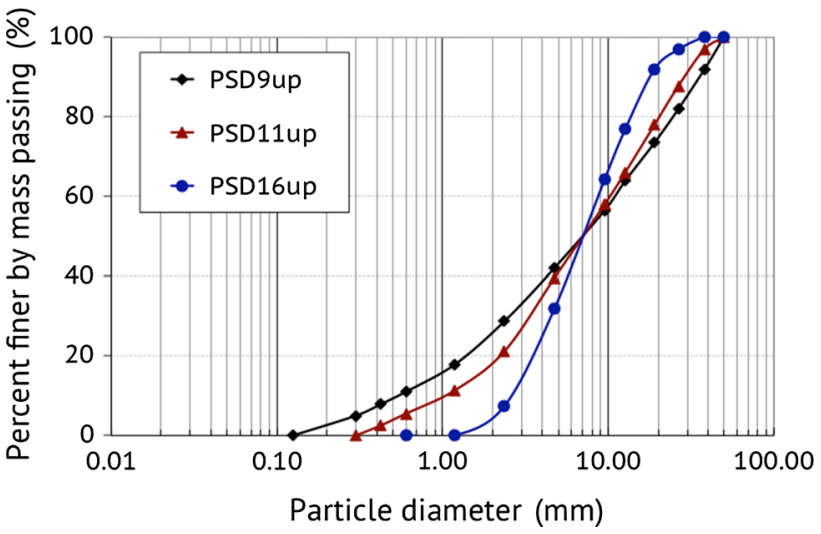

Fig. 3 Particle size distributions for the solid materials used in the tests

patches overlapping in the slope-normal direction up to the free surface of the flow. Several patch sizes were used, specifically $16 \times 16,24 \times 24,32 \times 32,40 \times 40$ and $48 \times 48$ pixels spaced at 8 pixels in each case. These correspond to spatial regions of $3.9 \times 3.9,5.8 \times 5.8,7.7 \times 7.7,9.6 \times 9.6$ and $11.5 \times 11.5 \mathrm{~mm}^{2}$ for $\mathrm{C}_{U}=3$ and $\mathrm{C}_{U}=10$ tests. For the tests with $\mathrm{C}_{\mathrm{U}}=20$, the spatial regions resulted as $3.0 \times 3.0,4.3 \times 4.3,5.8 \times 5.8,7.2 \times 7.2$ and $8.6 \times 8.6 \mathrm{~mm}^{2}$ because, due to the small thickness of the experimental flow in comparison to the other two PSDs (Fig. 4), the spatial resolution of the images was slightly increased by focussing the camera on a slightly smaller area.

The mean velocity profiles were obtained by averaging over 30 frames, at subsequent time steps, i.e. for a pair of consecutive frames over a time interval of $0.027 \mathrm{~s}$. The outliers were filtered calculating a trimmed mean by discarding the values falling outside a proper confidence interval. For patches of 16 pixels (hereafter called 16 pix), the confidence interval was set to one standard deviation in order to cope with the large scatter associated with the corresponding data, for all the others the confidence interval was set to two standard deviations. The evolution of the mean velocity with time obtained with the 16 pixels mesh is shown in Fig. 5. The 16 pix patch size is used only to assess flow height and velocity here. Due to the excessive noise, results are not reported for granular temperature [27] — see below. For patch sizes of 24 pixels and above, the measured granular temperature was calculated as, $T=\frac{1}{2}\left(\left\langle v_{x}^{\prime 2}+v_{y}^{\prime 2}\right\rangle\right)$, where $\mathrm{v}^{\prime}$ indicates the fluctuation component of the instantaneous velocity $v$, defined as $v^{\prime}=v-\langle v\rangle$, and $\langle\mathrm{v}\rangle$ is the mean velocity of the patch.

\section{Results}

\subsection{Flow height, velocity and fluctuation velocity}

The analysis starts immediately behind the unsaturated front where fully saturated conditions make the PLIF technique 
Fig. 4 Examples of test images with 48 and 24 pixels meshes. a During a test with $\mathrm{C}_{\mathrm{U}}=3$; b during a test with $\mathrm{C}_{\mathrm{U}}=20$. Overlapping patches of each mesh are centered at the same 8 pixel spacing and position. The patch at the top of each mesh is highlighted with thicker edges
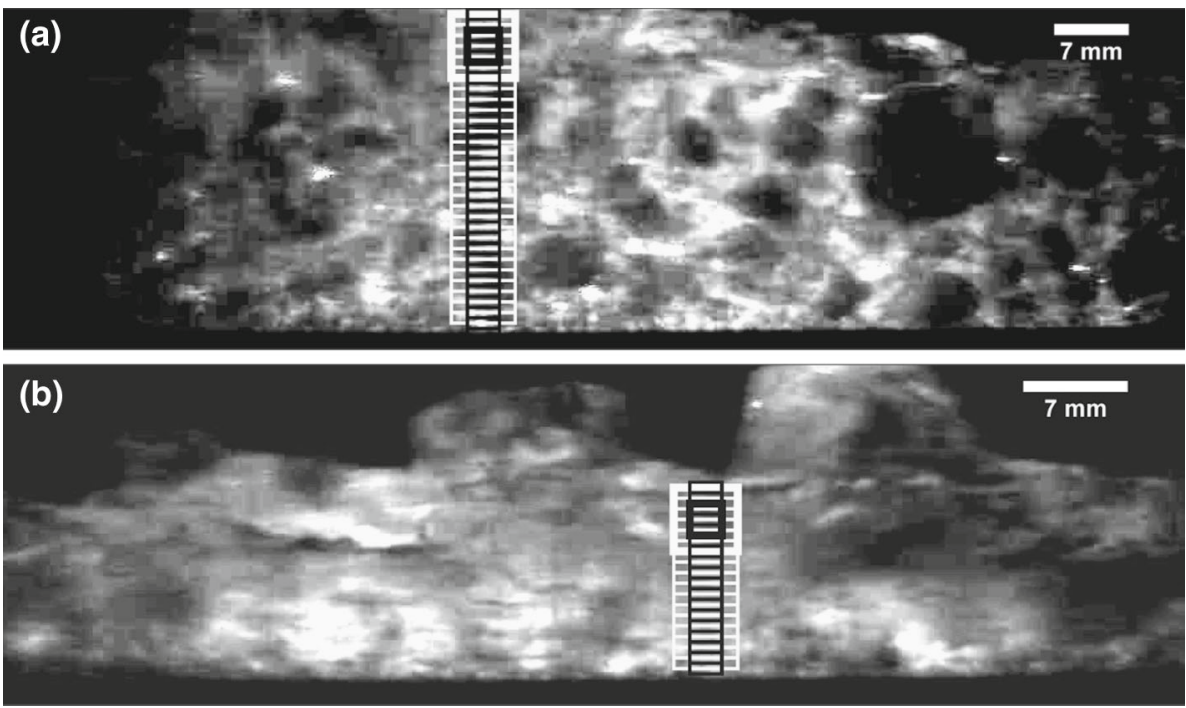

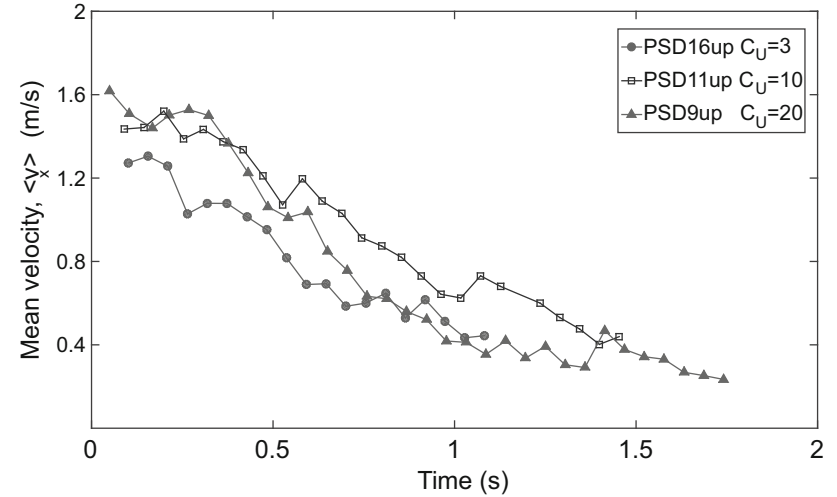

Fig. 5 Time evolution of the mean velocity estimated with 16 pixels mesh

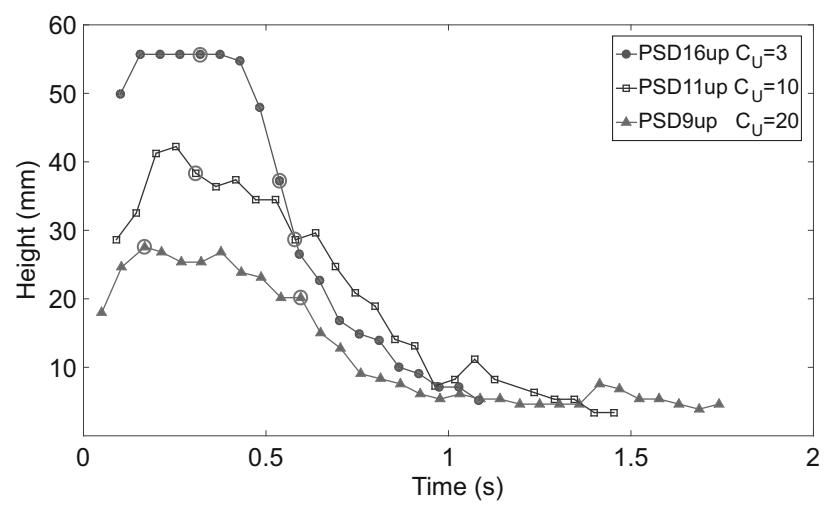

Fig. 6 Time evolution of flow heights (note truncation of flow height for PSD16 tests owing to flow thickness being larger than the image height). Circles indicate positions of meshes used for subsequent analyses

effective. In Fig. 6 the estimated heights of the experimental flows over time are plotted, using the position of the highest patch of each 16 pix mesh. This is actually an approximation of the true height, since the meshes cannot cover the entire depth of the flow given the mesh top is set just below the free surface. In the figure the position of the meshes adopted for the subsequent analyses (Figs. 7, 8, 9, 10,11) are highlighted with circles. For each test, one position close to the front and a second towards the tail of the surge were chosen to investigate the different behaviour exhibited by these segregating granular systems. Figure 7 shows the $\mathrm{x}$ and $\mathrm{y}$ components of the mean velocity as measured using the different PIV patch sizes for the three different PSDs. The y velocity component was found to be at least one order of magnitude smaller than the $\mathrm{x}$ component.

All the experimental flows were found to decelerate during the tests, and exhibit slip velocity at the base, although the streamwise velocity was generally higher for higher $C_{U}$ and the slip velocity was greater. It is notable that data from the 16 pixel patch fluctuates considerably, while the other data tend to collapse onto a similar trend of mean velocity. This suggests that the 16 pixel patch is beyond the minimum limit of acceptable size for the image texture (i.e. the spatial variation of brightness) to be tracked by the software. As patches become smaller, due to less information being available in each patch, the tracking is increasingly noisy. For instance, the 16 pixel patch size has a 2.25 times reduction of this information compared to the 24 pixel patch size. In Fig. 7, the standard deviation values of 24 and 48 pixels patches are also shown as error bars. Here, as expected, we see a greater standard deviation for 24 pixel patches than 48 .

In Fig. 8 the $x$-component of the velocity fluctuations, $v_{x}^{\prime}$, is presented. The y-component is not shown because its value can be strongly affected by the precision of the software. The measurements of $\mathrm{v}_{\mathrm{x}}^{\prime}$ at the front range from approximately $0.1-0.4 \mathrm{~m} / \mathrm{s}$, with larger values towards the top half part of the flow. Close the free surface, local high values are estimated (e.g. a spike in the $\mathrm{C}_{\mathrm{U}}=10$ test), due to the presence of the largest particles in this portion of the flow. This can cause 

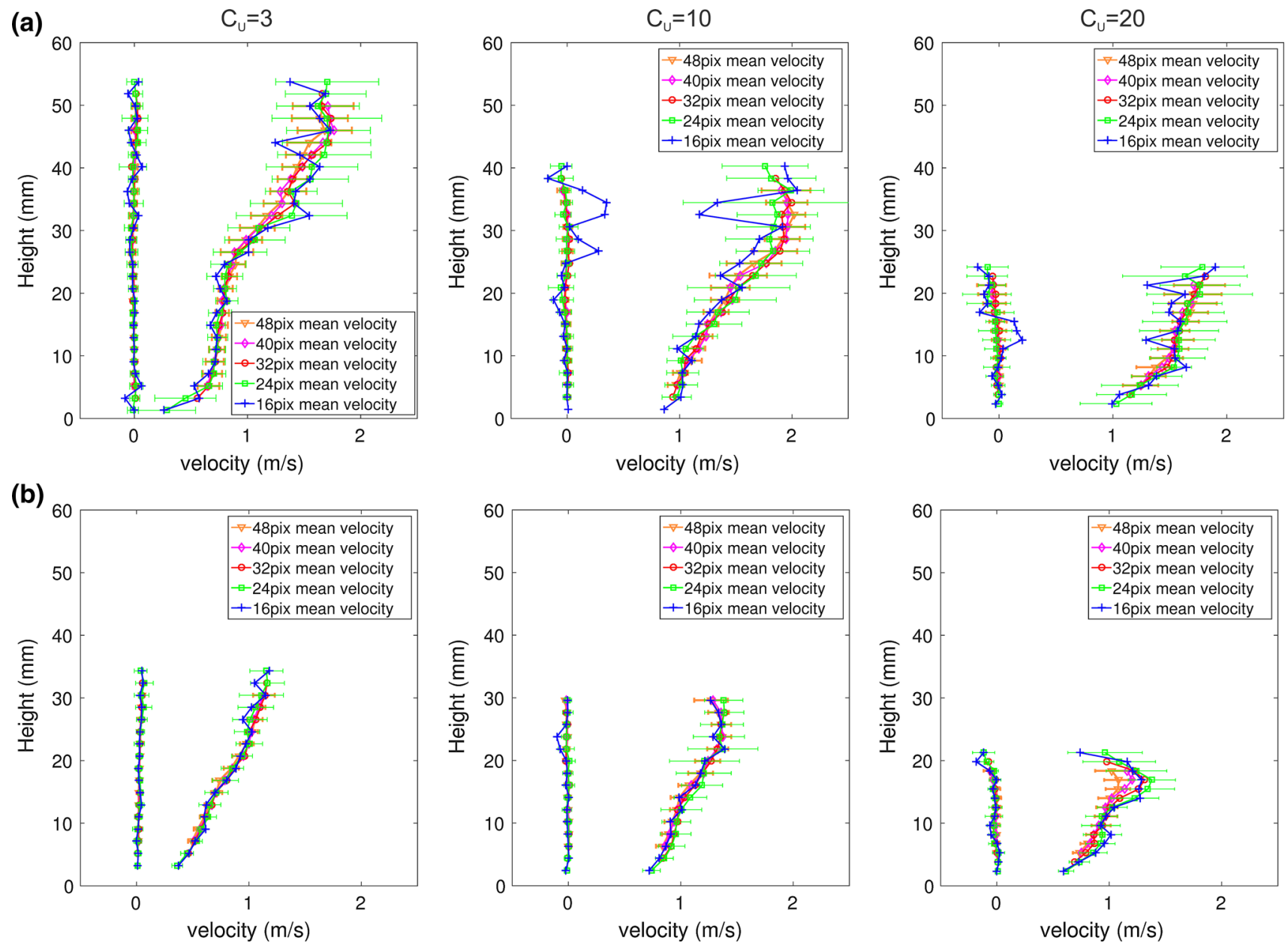

Fig. 7 Mean velocity estimated with each patch size. a Results for the meshes located towards the front; $\mathbf{b}$ results for the meshes located toward the tail of the flow. $\mathrm{v}_{\mathrm{x}}$ is on the right and $\mathrm{v}_{\mathrm{y}}$ is on the left in each panel. The standard deviation values (error bars) of 24 and 48 pixels patches are also shown

The measurements for the flow tail (Fig. 8b) show slightly higher fluctuations for the higher $\mathrm{C}_{\mathrm{U}}$ tests, but otherwise broadly similar behaviour for all of the tests with fluctuations generally increasing toward the surface. This is possibly due to the fact that the composition of the mixture towards the tail is more similar than at the front because in segregating systems most of the large particles have focused to the front of the flow once the tail has developed.

\subsection{Granular temperature}

Reynolds et al. [34] presented a method to measure granular temperature for steady granular flows using a PIV based method, and examined the influence of PIV patch size on the determined granular temperature of a uniform dry granular mixture under shear in a granulator. In their experiments, the granular temperature measured using a PIV patch or interrogation size equivalent to the particle size was considered 

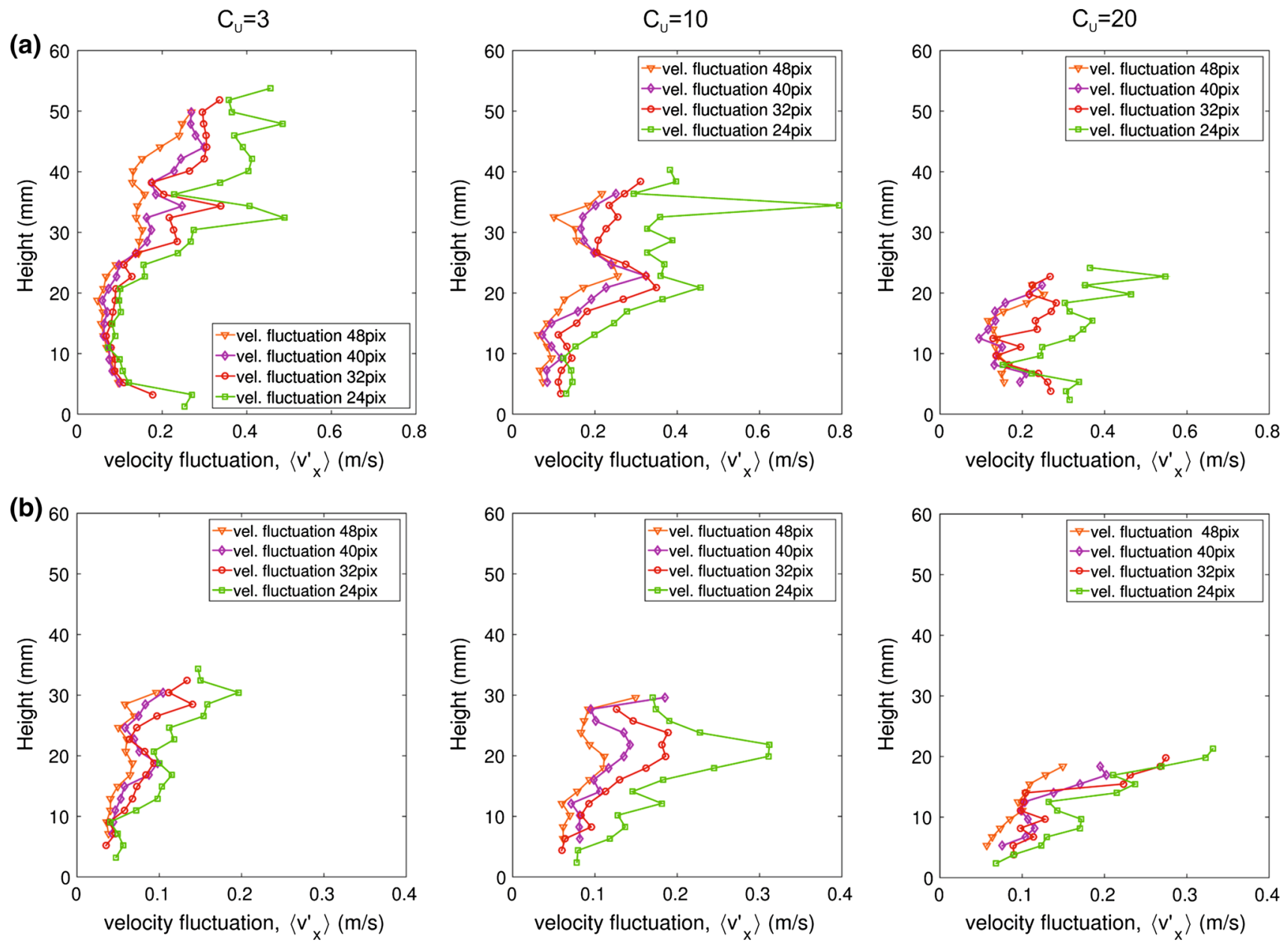

Fig. 8 Velocity fluctuations estimated with each patch size. a Results for meshes located towards the front; $\mathbf{b}$ results for meshes located toward the tail of the flow

to represent the true granular temperature. They found that, assuming no spatial correlation between the patches (i.e. with truly random fluctuations in velocity from the mean) and with changes in granular temperature being small over the region of observation, as the interrogation area was increased by a factor $\mathrm{n}$, the measured granular temperature reduced by the same factor $n$. Use of this fact would have the advantage of enabling the true granular temperature to be inferred from larger patches (i.e. a coarser PIV analysis), with an attendant increase in the precision of correlation and hence a reduction in error. In this study, we examine whether this approach may be used to examine the granular temperature of our unsteady, well graded, granular systems. Here, we take 24 pixel patches as being the minimum size for comparison. This is equivalent to $7.7 \mathrm{~mm}$ (similar to mean particle size) for $\mathrm{C}_{\mathrm{U}}$ of 3 and 10 , and $5.8 \mathrm{~mm}$ (slightly smaller than mean) for $\mathrm{C}_{U}$ of 20 . Hence for results determined using 32 pixel patches, $\mathrm{n}$ for scaling comparison will be $(32 / 24)^{2}=1.778$, and so on.

Figure 9 shows, for all the PSDs, the granular temperatures, obtained with the various patch sizes, scaled by factor $\mathrm{n}$ with reference to the 24 pixel patch. At the front, the data from $\mathrm{C}_{\mathrm{U}}=3$ is seen to scale quite well-i.e. the data generally falls on a single curve, with some deviation in the upper portion of the flow. Data for $\mathrm{C}_{\mathrm{U}}=10$ still scale although with larger dispersion. $\mathrm{C}_{\mathrm{U}}=20$ exhibits still greater dispersion, although the rule does appear to result in collapse of the data to the same order in terms of T. This may be interpreted as the data being spatially uncorrelated for the uniform flow $\mathrm{C}_{\mathrm{U}}=3$, while for $\mathrm{C}_{\mathrm{U}}=10$ and especially $\mathrm{C}_{\mathrm{U}}=20$ the flow being actually somewhat correlated (i.e. fluctuations are not completely random) at the scale in question. To check this, it is possible to define a spatial correlation function $\rho_{\mathrm{ij}}$ between the temporal velocities of the patches at position $i$ and $j$ in the mesh, as follows:

$\rho_{i, j}=\frac{\sigma_{i, j}}{\sigma_{i} \sigma_{j}}$

where $\sigma_{i, j}$ is the covariance of the velocity $\mathrm{i}$ and $\mathrm{j}$ and $\sigma_{i}$ and $\sigma_{j}$ their standard deviation (more information can be found in Reynolds et al. [34]). If there is no spatial correlation: 

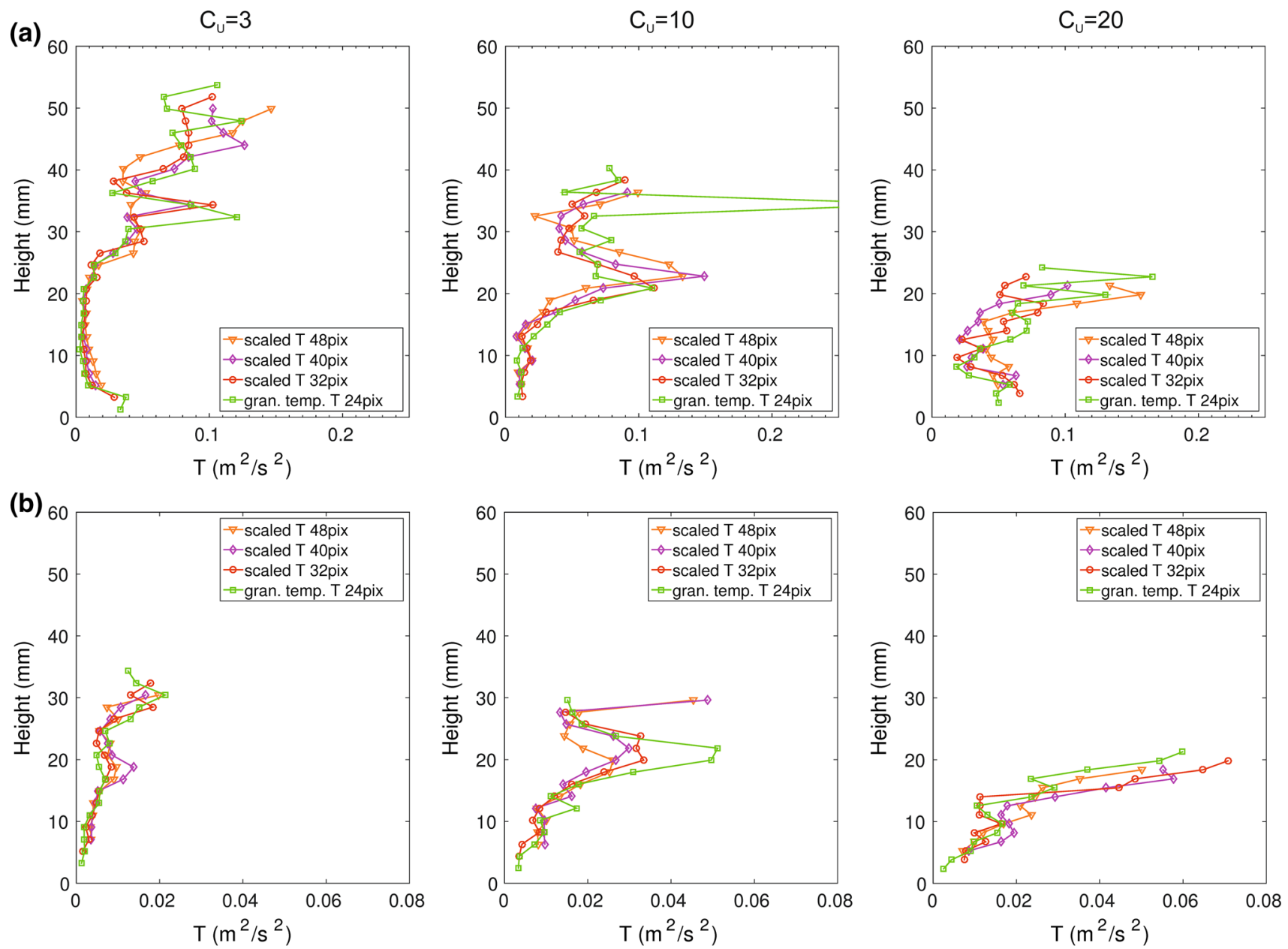

Fig. 9 Scaled granular temperature according to patch size: a results for the meshes located towards the front; $\mathbf{b}$ results for the meshes located toward the tail of the flow

$\rho_{i, j}= \begin{cases}1 & i=j \\ 0 & i \neq j\end{cases}$

Figure 10 shows the resulting spatial correlation calculated at the front for the test with $\mathrm{C}_{\mathrm{U}}=3$ and $\mathrm{C}_{\mathrm{U}}=20$ using the mesh with 32 pixels size. For clarity, in the figure only the results for three patches are displayed for each mesh: close to the free surface; in the middle of the flow; towards the bottom of the flume. The patch number indicates the position of the patches normal to the flow depth with numbering starting at the top of the mesh (i.e. the top of the flow). All the patches show large values of correlation coefficient within a distance of three patches from the considered point (i.e. the velocities of the adjoining patches are correlated). This is due to the fact that the patches positions are overlapped with the spacing being $1 / 4$ of the patch size. Beyond this distance, for the test with $\mathrm{C}_{U}=3$ the velocities of the patches are poorly correlated, that is, $\rho$ is always much smaller than 0.4 and fluctuating around 0 . The $\mathrm{C}_{\mathrm{U}}=20$ test shows analo- gous behaviour for the patches at the top of the mesh, with relatively poor correlation along the depth. However, higher correlation is found among the patches located towards the bottom half, with the correlation coefficient increasing from a value of $\sim 0.4$ to 0.8 . This can be explained by the nature of $\mathrm{C}_{\mathrm{U}}=20$ having particles of comparable size to the flow thickness, whose influence increases the correlation length scale. That is larger particles pushing through the flowbouncing and colliding with the base may drag or shunt the particles around them generating a correlated motion along the depth. Towards the tail, all the PSDs show improved scaling, confirming that in this part of the flow the behaviour of the material is more regular and less disrupted by the segregation process and the presence of larger particles.

\section{Discussion}

Previous researchers examining granular free-surface flows have measured granular temperature in both two and three- 
Fig. 10 Coefficient of spatial correlation calculated for three patches of the 32 pixels mesh at the front of the test: a $\mathrm{C}_{\mathrm{U}}=3$ and $\mathbf{b} \mathrm{C}_{\mathrm{U}}=20$
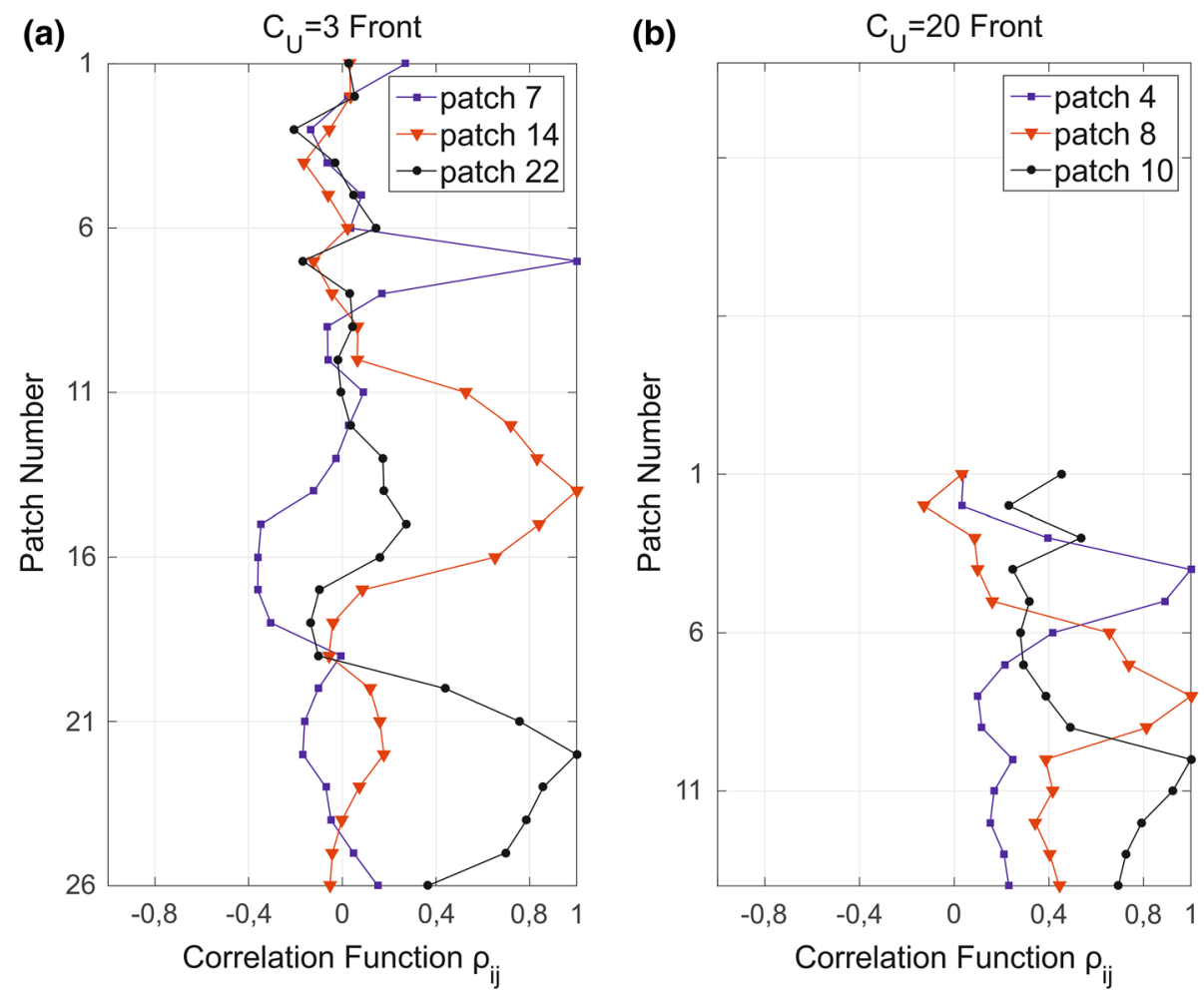
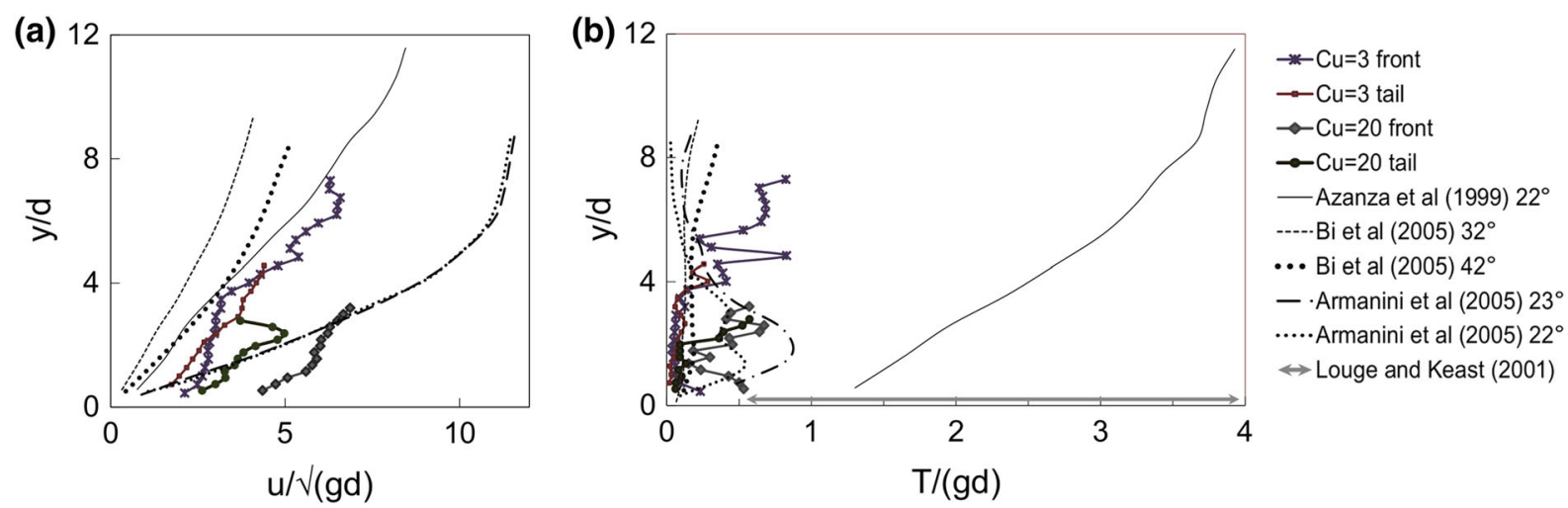

Fig. 11 Relationship between a normalised flow height against normalised velocity; $\mathbf{b}$ normalised flow height against granular temperature

dimensional experiments. However, almost all reported flume-type experiments have been carried out using particles of near-uniform (monodisperse) size and, to our knowledge, all have been carried out on steady flows at the flow margins. It is worth examining how the results of such experiments compare with the present ones on unsteady and segregating flows with measurements taken interior to the flows.

Results are plotted in Fig. 11a as normalised velocity against normalised flow height and Fig. $11 \mathrm{~b}$ as granular temperature against normalised flow height. Data from this work for $\mathrm{C}_{U}=3$ and $\mathrm{C}_{U}=20$ are plotted for the front and tail profiles while $\mathrm{C}_{U}=10$ is omitted for clarity. Particle size has been taken as $\mathrm{D}_{50}$ to normalise the velocity and granular temperature profiles, in keeping with common practice. If a larger or smaller particle size was used, this would cause the plots to shrink or grow on the y-abscissa in comparison, highlighting the difficulties in ascribing a correct length scale for polydisperse flows. Data from three other test arrangements are plotted for comparison. Two sets of $2 \mathrm{D}$ experiments $[11,12]$ highlight how the granular temperature-and hence flow regime - can be altered by relatively subtle changes in slope angle, with an increasing gradient resulting in more energetic and collisional flows. Azanza et al. [11] carried out 2D flow experiments using dry $3 \mathrm{~mm}$ metallic beads in the collisional-kinetic regime, finding normalised temperatures of between 1 and 4 for fully collisional flows and higher values again for the kinetic (highly dispersed) regime. A single representative collisional flow is plotted in Fig. 11. There appears to be a slight slip velocity at the base if the data is extrapolated downward, while granular temperature 
increases steadily towards the surface. Bi et al. [12] carried out 2D experiments on frictional-collisional flows using $8 \mathrm{~mm}$ diameter polystyrene disks which were marked in such a way as to enable the determination of the disk rotation. In this way they partitioned granular temperature into slope normal and parallel translational components and a rotational component. Normalised temperatures were found of up to 0.4 but mainly in the order of 0.1 . The stream-wise velocity and granular temperature profiles for one representative flow are plotted in Fig. 11, showing a no-slip condition at the base and a somewhat non-steady granular temperature with depth. They report that preliminary tests on polydisperse flows gave slightly higher granular temperatures which they attributed to segregation.

In terms of 3D experiments, Louge and Keast [14] have reported normalised basal granular temperature values of between 0.55 and 3.96 for experimental 3D flows of $3 \mathrm{~mm}$ spherical dry glass beads over a flat frictional surface-again showing a large range of values. Because no profiles were reported, these values are indicated as a range in Fig. 11. Armanini et al. [10], in one of the few experimental suites to examine flow of liquid-granular mixtures, conducted conveyor flume tests using PVC pellets of $3.7 \mathrm{~mm}$ equivalent diameter and water. Figure 11 plots two representative tests from flows over a solid bed, which most closely approximate the situation examined in this paper. For such tests, while velocity profiles are almost identical, normalised granular temperature are seen to vary from test to test, with local values ranging from approximately $0.1-1.0$. One notable feature is that the granular temperature profiles are not monotonic, and increase from the base rapidly before decreasing gradually towards the surface. This feature is also seen in the shear rate, so the two appear to be correlated, as seen in other work $[13,35,36]$. From these cases, it may be seen that while normalised flow velocity profiles are often quite similar for different 2D and 3D arrangements (Fig. 11a), there can be differences in granular temperature of an order of magnitude (Fig. 11b). In terms of material properties alone, this can be attributed to differences in the particle coefficient of restitution [37] (noting that the metal and glass particle flows result in higher $\mathrm{T}$ than those using PVC and polystyrene), solid fraction, presence or otherwise of viscous fluid (resulting in both viscous effects and the development of fluid pore pressure) and polydispersity. In addition external experimental arrangements, such as flow width, depth, mass, slope and basal friction can all have an influence on the granular temperature profile.

In terms of velocity profiles, the results reported here on unsteady flows are, unsurprisingly, less monotonic than those from steady flows, and show greater basal slip, particularly at the front and for higher $\mathrm{C}_{\mathrm{U}}$, which as previously stated appears more as a plug-flow. The greater slip can be attributed to higher polydispersity [6] and the fact that these measure- ments are not affected by flume corners [29]. Otherwise, the profiles are quite similar with height above the base. Regarding granular temperature, the data differ according to $\mathrm{C}_{\mathrm{U}}$. Specifically, the data for $\mathrm{C}_{U}=3$ show very low values at the base as discussed, increasing toward the surface, while for $\mathrm{C}_{U}=20$ the data shows large values throughout the flow, unsteadily increasing with height above the base but otherwise producing granular temperature values similar to those of Armanini et al. [10].

It may be seen from Fig. 7 that the greatest shear rate (i.e. velocity gradient with depth) for the flows is found towards the base, and for $C_{U}=20$ the shear rate is nearly negligible in the top third of the flow (i.e. plug-flow). One might expect that the greatest granular temperature would therefore occur near the flow base and in other zones of greatest shear rate as seen in other work $[10,13]$ where solids concentration is either constant with depth or increases vertically. Instead, we see that the measured $\mathrm{T}$ is highest in this upper zone. Given that as solid concentration reduces, fluctuation velocity and hence granular temperature tends to increase [9], the implication is that the solid concentration at the surface is less than towards the base. While this is not directly measured, it can be seen to an extent in the video images. However, this behaviour is rather different from observations from [10] and according to numerical simulations of dry steady uniform flows [23] in which solid concentration increases towards the free surface for a relatively smooth bed-i.e. where a slip velocity is seen. The combined influence of both fluid and relatively polydisperse particles may be responsible for this departure. Fine particles dominating the lower flow regions will increase local viscosity and pore pressure, reducing collisional stress transfer and reducing $\mathrm{T}$ near the base. Larger particles pushing through the flow-bouncing and colliding with the base will disrupt this behaviour and may lead to the observed $\mathrm{T}$ being greater throughout the flow. Toward the tail fluctuations are lower than those estimated close to the front but slightly higher for higher $\mathrm{C}_{\mathrm{U}}$. This is likely to be due to the residual presence of a few large particles in the tail of the well-graded flows that can enhance the collisions there and generally disrupt the flow.

The tests conducted here highlight some important differences between uniform and well-graded flows. Well graded flows segregate more and exhibit more plug-like flow globally, while at the same time, velocity fluctuations are distributed over the entire depth and appear to be unrelated to shear rate. However in the analysis for the well graded flows it should be noted that the estimates of the velocity fluctuations can be affected by random errors where particles with size much larger than the patch size are present [33]. In the case of $C_{U}=20$, particles of size $D_{90}$ or larger can be at least as large as the flow thickness-which also is what is found to occur in nature [38]. 


\section{Conclusions}

In this work the interior instantaneous velocity fields within physical models of debris flow were investigated via Particle Image Velocimetry. A series of experiments were conducted in a small-scale flume using three different particle size distributions (PSDs) with the same mean sediment volume concentration and mean particle size $\mathrm{D}_{50}$. Two-dimensional images of the resulting unsteady and segregating flows over rigid beds were recorded at a section located close the end of the chute, within the flow, away from sidewall influences. For each test the analysis were performed in one position close to the front and a second towards the tail of the surge in order to investigate the range in behaviour exhibited by these granular systems.

The effect of PSD and PIV patch size on the estimation of velocity fluctuations were examined. The velocity fluctuations were found, for all the tests, to be higher at the front of the flow and close to the free surface. However the estimates of the velocity fluctuations towards the free surface can be affected by random errors due to the presence of particles with size much larger than the patch size used. The behaviour towards the bottom changes, with lower fluctuations observable halfway down in $\mathrm{C}_{\mathrm{U}}=3$, and in the bottom third in $\mathrm{C}_{U}=10$, while $\mathrm{C}_{U}=20$ shows large fluctuation values along most of the depth. At the tail the fluctuations were found slightly higher for the higher $\mathrm{C}_{U}$ but for all the tests they were found generally increasing toward the surface.

A relationship proposed by Reynolds et al. [34] using PIV to calculate the granular temperature data at different scales of scrutiny was applied to our results. The method relies on the data not being spatially correlated. This approach was found to give consistent results for the relatively uniform particle size distribution but those for the well-graded material showed less good agreement, especially at the front of the flow. To investigate this, the degree of correlation with depth was investigated using 32 pixel interrogation patches for flows with $\mathrm{C}_{U}=3$ and $\mathrm{C}_{U}=20$. We found little correlation (beyond patch overlap) throughout the flow for the uniform material, but somewhat correlated motion, particularly in lower part of the flow, for the well graded material. This suggests that in order to determine the granular temperature for well-graded flows, larger interrogation patch sizes should be used. However larger patches for these unsteady flows can be of the order of the flow thickness which may preclude such an approach. This study shows that while PIV may offer promise for the measurement of granular temperature in flowing granular systems, practically speaking, it may be limited to being applied to relatively uniform particle gradings and/or deep flows.

Open Access This article is distributed under the terms of the Creative Commons Attribution 4.0 International License (http://creativecomm ons.org/licenses/by/4.0/), which permits unrestricted use, distribution, and reproduction in any medium, provided you give appropriate credit to the original author(s) and the source, provide a link to the Creative Commons license, and indicate if changes were made.

\section{References}

1. Hungr, O.: Classification and terminology. In: Jakob, M., Hungr, O. (eds.) Debris-flow hazards and related phenomena. Springer Praxis Books. Springer, Berlin (2005)

2. Midi, G.D.R.: On dense granular flows. Eur. Phys. J. E 14, 341-369 (2004)

3. Takahashi, T.: Debris Flow. IAHR Monograph Series. A. A. Balkema, Rotterdam (1991)

4. Johnson, C.G., Kokelaar, B.P., Iverson, R.M., Logan, M., LaHusen, R.G., Gray, J.M.N.T.: Grain-size segregation and levee formation in geophysical mass flows. J. Geophys. Res. 117, F01032 (2012). doi:10.1029/2011JF002185

5. Berger, C., McArdell, B.W., Schlunegger, F.: Direct measurement of channel erosion by debris flows, Illgraben, Switzerland. J. Geophys. Res. 116, F01002 (2011). doi:10.1029/2010JF001722

6. Rognon, P.G., Roux, J.N., Naaim, M., Chevoir, F.: Dense flows of bidisperse disks down an inclined plane. Phys. Fluids 19(5), 058101 (2007)

7. Campbell, C.S.: Granular shear flows at the elastic limit. J. Fluid Mech. 465, 261-291 (2002). doi:10.1017/S002211200200109X

8. da Cruz, F., Emam, S., Prochnow, M., Roux, J.-N., Chevoir, F.: Rheophysics of dense granular materials: discrete simulation of plane shear flows. Phys. Rev. E 72, 021309 (2005)

9. Ahn, H., Brennen, C.E., Sabersky, R.H.: Measurements of velocity, velocity fluctuation, density, and stresses in chute flows of granular materials. Trans. ASME J. Appl. Mech. 58(3), 792 (1991)

10. Armanini, A., Capart, H., Fraccarollo, L., Larcher, M.: Rheological stratification in experimental free-surface flows of granular-liquid mixtures. J. Fluid Mech. 532, 269-319 (2005)

11. Azanza, E., Chevoir, F., Moucheront, P.: Experimental study of collisional granular flows down an inclined plane. J. Fluid Mech. 400, 199-227 (1999). doi:10.1017/S0022112099006461

12. Bi, W., Delannay, R., Richard, P., Valance, A.: Experimental study of two-dimensional, monodisperse, frictional-collisional granular flows down an inclined chute. Phys. Fluids 18, 123302 (2006). doi:10.1063/1.2405844

13. Campbell, C.S., Brennen, C.E.: Chute flows of granular material: Some computer simulations. Trans. ASME J. Appl. Mech. 52, 172 178 (1985)

14. Louge, M.Y., Keast, S.C.: On dense granular flows down flat frictional inclines. Phys. Fluids 13, 1213 (2001). doi:10.1063/1. 1358870

15. Bagnold, R.A.: Experiments on a gravity-free dispersion of large solid spheres in a Newtonian fluid under shear. Proc. R. Soc. Lond. Ser. A 225, 49-63 (1954)

16. Iverson, R.M.: The physics of debris flows. Rev. Geophys. 35(3), 245-296 (1997) 
17. Savage, S.B., Hutter, K.: The motion of a finite mass of granular material down a rough inclined plane. J. Fluid Mech. 199, 177-215 (1989)

18. Iverson, R.M., Vallance, J.W.: New views of granular mass flows. Geology 29(2), 115-118 (2001)

19. Iverson, R.M.: Scaling and design of landslide and debris-flow experiments. Geomorphology (2015). doi:10.1016/j.geomorph. 2015.02.033

20. Phillips, C.J., Davies, T.R.H.: Determining rheological parameters of debris flow material. Geomorphology 4, 101-110 (1991)

21. Zhou, G.G.D., Ng, C.W.W.: Dimensional analysis of natural debris flows. Can. Geotech. J. 47(7), 719-729 (2010). doi:10.1139/ T09-134

22. Ogawa, S.: Multi-temperature theory of granular materials. Paper Presented at the US-Japan Seminar on Continuum-Mechanical and Statistical Approaches in the Mechanics of Granular Materials, Sendai, Japan, 5-9 June 1978

23. Campbell, C.S.: Rapid granular flows. Annu. Rev. Fluids 22, 57-92 (1990)

24. Remy, B., Khinast, J.G., Glasser, B.J.: Polydisperse granular flows in a bladed mixer: experiments and simulations of cohesionless spheres. Chem. Eng. Sci. 66, 1811-1824 (2011)

25. Deen, N.G., Dijkhuizen, W., Bokkers, G.A., van Sint Annaland, M., Kuipers, J.A.M.: Validation of the granuar temperature prediction of the kinetic theory of granular flow by particle image velocimetry and a discrete particle model. Paper Presented at the 3rd International Symposium on Two-Phase Flow Modelling and Experimentation, Pisa, 22-24 Sept 2004

26. Dijkhuizen, W., Bokkers, G.A., Deen, N.G., van Sint Annaland, M., Kuipers, J.A.M.: Extension of PIV for measuring granular temperature field in dense fluidized beds. AIChE J. 53(1), 108-118 (2007). doi:10.1002/aic.11044

27. Sanvitale, N., Bowman, E.T.: Towards measurement of "granular temperature" inside a flowing unsteady saturated granular medium. Paper Presented at the ISSMGE International Symposium on Geomechanics from Micro to Macro, Cambridge 1-3 Sept 2014
28. Sanvitale, N., Bowman, E.T.: Internal imaging of saturated free surface flows. Int. J. Phys. Model. Geotech. 12(4), 129-142 (2012). doi:10.1680/ijpmg.12.00002

29. Bird, J.J., Bowman, E.T.: A comparison of internal and external flow behaviour of laboratory-scale debris flows. Paper Presented at the ICPMG2014 -8th International Conference on Physical Modelling in Geotechnics, Perth

30. Hassan, Y.A., Dominguez-Ontiveros, E.: Flow visualization pebble bed reactor experiment using PIV and refractive index matching techniques. Nucl Eng Des. 238(11), 3080-3085 (2008)

31. White, D.J., Take, W.A., Bolton, M.D.: Soil deformation measurement using particle image velocimetry (PIV) and photogrammetry. Géotechnique 53(7), 619-631 (2003)

32. Bryant, S.K., Take, W.A., Bowman, E.T.: Observations of grainscale interactions and simulation of dry granular flows in a largescale flume. Can. Geotech. J. 52, 638-655 (2015). doi:10.1139/ cgj-2013-0425

33. Wilson, B.M., Smith, B.L.: Uncertainty on PIV mean and fluctuating velocity due to bias and random errors. Meas. Sci. Technol 24, 035302 (2013). doi:10.1088/0957-0233/24/3/035302

34. Reynolds, G.K., Nilpawar, A.M., Salman, A.D., Hounslow, M.J.: Direct measurement of surface granular temperature in a high shear granulator. Powder Technol. 182, 211-217 (2008)

35. Losert, W., Bocquet, L., Lubensky, T.C., Gollub, J.P.: Particle dynamics in sheared granular matter. Phys. Rev. Lett. 85(7), 1428$1431(2000)$

36. Mahmood, Z., Dhakal, S., Iwashita, K.: Measurement of particle dynamics in rapid granular shear flows. J. Eng. Mech. 135, 285-294 (2009)

37. Marinack Jr., M.C., Jasti, V.K., Choi, Y.E., Higgs Iii, C.F.: Couette grain flow experiments: the effects of the coefficient of restitution, global solid fraction, and materials. Powder Technol. 211(1), 144155 (2011). doi:10.1016/j.powtec.2011.04.012

38. McArdell, B., Bartelt, P., Kowalski, J.: Field observations of basal forces and fluid pore pressure in a debris flow. Geophys. Res. Lett. 34, L07406 (2007). doi:10.1029/2006GL029183 